\title{
Kalman filter-based prediction refinement and quality enhancement for geometry-based point cloud compression
}

\author{
Lu Wang \\ School of Info. Sci. and Eng. \\ Shandong University \\ Qingdao, China \\ lu_wang@mail.sdu.edu.cn
}

\author{
Jian Sun (corresponding author) \\ School of Info. Sci. and Eng. \\ Shandong University \\ Qingdao, China \\ sunjian@sdu.edu.cn
}

\author{
Hui Yuan \\ School of Contr. Sci. and Eng. \\ Shandong University \\ Jinan, China \\ huiyuan@sdu.edu.cn
}

\author{
Raouf Hamzaoui \\ School of Eng. and Sustain. Dev. \\ De Montfort University \\ Leicester, UK \\ rhamzaoui@dmu.ac.uk
}

\author{
Xiaohui Wang \\ School of Info. Sci. and Eng. \\ Shandong University \\ Qingdao, China \\ sduwangxiaohui@hotmail.com
}

\begin{abstract}
A point cloud is a set of points representing a three-dimensional (3D) object or scene. To compress a point cloud, the Motion Picture Experts Group (MPEG) geometrybased point cloud compression (G-PCC) scheme may use three attribute coding methods: region adaptive hierarchical transform (RAHT), predicting transform (PT), and lifting transform (LT). To improve the coding efficiency of PT, we propose to use a Kalman filter to refine the predicted attribute values. We also apply a Kalman filter to improve the quality of the reconstructed attribute values at the decoder side. Experimental results show that the combination of the two proposed methods can achieve

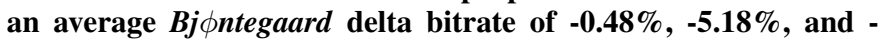
6.27\% for the Luma, Chroma $\mathrm{Cb}$, and Chroma $\mathrm{Cr}$ components, respectively, compared with a recent G-PCC reference software.
\end{abstract}

Index Terms-point clouds, predictive coding, Kalman filter

\section{INTRODUCTION}

With the rapid development of 3D sensing technologies, such as laser scanning and oblique photogrammetry, point clouds have become an important source of data [1] [2]. Highprecision point clouds are widely used in 3D digital modeling of smart cities, autonomous driving, heritage protection, etc. Due to the huge data volume of point clouds, efficient compression is critical.

To compress point clouds, MPEG proposed a video-based compression platform (V-PCC) [3] and a geometry-based compression platform (G-PCC) [4]. Compared to V-PCC, GPCC is more appropriate for sparse point clouds [5]. In this paper, we focus on G-PCC.

G-PCC can use three transforms for coding the attribute information of the points: region adaptive hierarchical transform (RAHT) [6], predicting transform (PT) [7], and lifting transform (LT) [8]. The latter two are based on Level of Details (LODs) [7] as shown in Fig. 1. Using a set of fixed distances $\left(d_{l}\right)_{l=0 \ldots L-1}$ specified by the user, the point cloud is first re-organized into a set of refinement levels $\left(R_{l}\right)_{l=0 \ldots L-1}$. Then, the $l$ th level of detail, denoted by $\operatorname{LOD}_{l}$, is obtained by taking the union of the refinement levels $R_{0}, R_{1}, \ldots, R_{l}$ (i.e., $\mathrm{LOD}_{l}=\mathrm{U}_{i=0}^{l} R_{i}$ ). Next, multiple predictor candidates for the current coding point are selected based on its $k$ nearest neighbors. The best predictor is finally determined with ratedistortion optimization [4]. Points in an LOD are predicted successively according to the generating order of LOD. Then the residuals are calculated as the difference between the original attribute and the predicted attribute and quantized before entropy coding. Since the reconstructed attribute of the current point is used to predict the attribute of the subsequent points, prediction errors may propagate and accumulate. An overview of the point cloud attribute compression procedure for PT is illustrated in Fig. 2. Based on the point-bypoint prediction structure of PT in G-PCC, we propose a Kalman filter-based prediction refinement method to improve the prediction accuracy and also propose a Kalman filter-based quality enhancement method for the reconstructed point cloud after decoding.

The remainder of the paper is organized as follows. Section II discusses related work. Section III presents the proposed method. Experimental results and conclusions are given in Section IV and V, respectively.

\section{RELATED WORK}

The architecture of G-PCC is shown in Fig. 3. In the encoder, point positions (geometry information) are coded first. Then, the attributes are coded based on the reconstructed geometry. For PT-based attribute coding, the Manhattan or the Euclidean distance is used to generate the LOD order and select the best predictor for each point in an LOD. Compared 


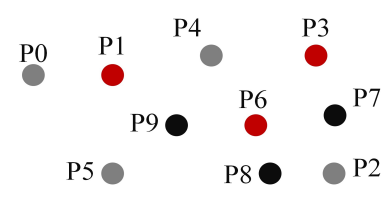

Original order

P0, P1, P2, P3, P4 ,P5, P6, P7, P8, P9

LOD-based order

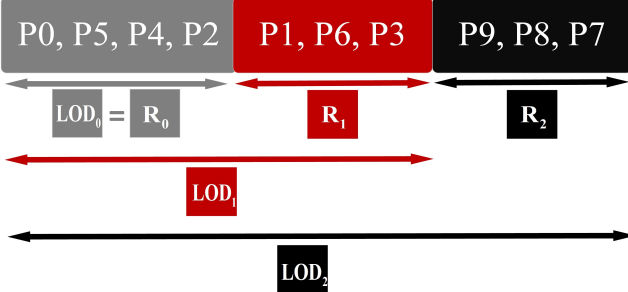

Fig. 1. LOD generation in G-PCC

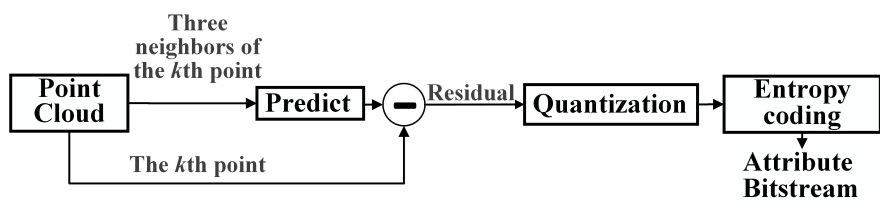

Fig. 2. Overview of attribute compression for PT.

with RAHT and LT, the prediction accuracy of PT still needs to be improved. Yea et al. [9] applied the bilateral filter as an in loop-filter for PT. Sugio et al. [10] proposed to select the best predictor with rate-distortion optimization. Ma et al. [11] proposed to smooth the attributes of neighbouring points before prediction. Zhang et al. [12] added a modification parameter called intraPredictionWeight to set the optimal weights of neighbouring points.

To further improve the coding efficiency as well as improve the reconstruction quality of point clouds, post-processingbased quality enhancement is also important. The existing point cloud enhancement methods are mainly aimed at geometric distortion. Alexa et al. [13] used the points at the vertices of the Voronoi diagram to interpolate in the local tangent space to complete the upsampling. Lipman et al. [14] proposed a point cloud upsampling method based on the locally-optimal-projection operator for surface reconstruction. However, there is no research focusing on enhancing the reconstruction quality of the attribute.

In this paper, we proposed to use a Kalman filter to improve the prediction accuracy during the encoding procedure and to improve the reconstruction quality of attributes after decoding.

\section{PRoposed METHOD}

In this section, we first provide the basic principles of the Kalman filter and then describe the details of the proposed method.

\section{A. Kalman filters}

The Kalman filter [15] is an efficient recursive filter. It can reduce the system prediction error gradually and is particularly suitable for stationary random signals. The Kalman filter uses

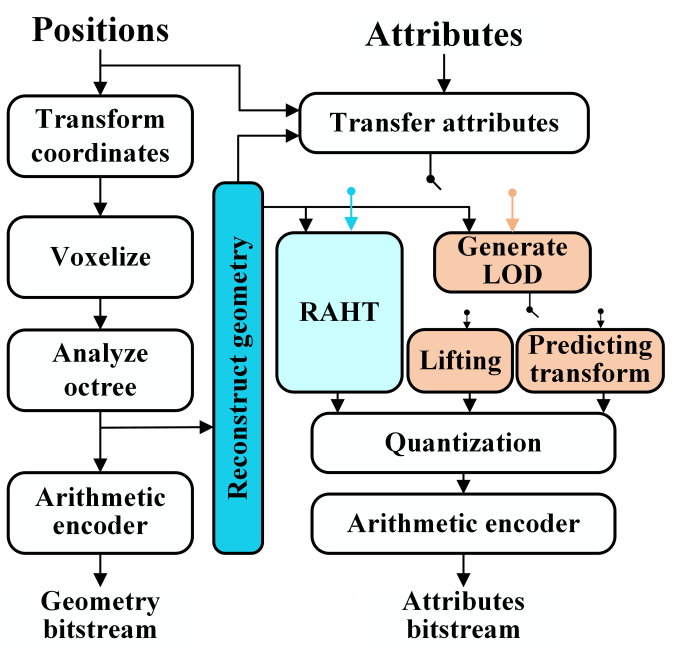

Fig. 3. Framework of G-PCC encoder.

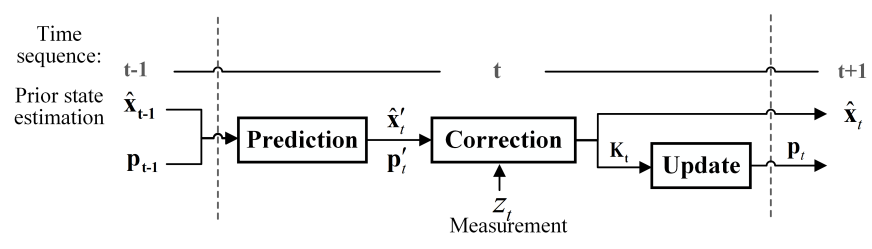

Fig. 4. Basic Kalman filter framework.

an estimation of the previous state to find the optimal value of the current state. As shown in Fig. 4, the Kalman filter consists of three main parts: prediction, correction and update. It requires two important input variables: the measured value $z_{t}$ and the initial prediction value $\widehat{x}_{t}^{\prime}$.

a) Prediction process: The initial prediction $\widehat{x}_{t}^{\prime}$ of the signal value $\widehat{x}_{t}$ at time $t$ can be represented by a linear function of the previous signal value $\widehat{x}_{t-1}$, i.e.,

$$
\widehat{\boldsymbol{x}}_{\boldsymbol{t}}^{\prime}=\mathbf{A} \widehat{\boldsymbol{x}}_{t-1}+\boldsymbol{w}_{t-1},
$$

where $\boldsymbol{A}$ is the state transition matrix (usually an identity matrix) and $\boldsymbol{w}_{\boldsymbol{t}-\mathbf{1}}$ represents noise.

To calculate the Kalman gain (the minimum variance between the original and the predicted values) and correct the initial prediction, the prediction error covariance matrix $\boldsymbol{P}_{\boldsymbol{t}}^{\prime}$ of

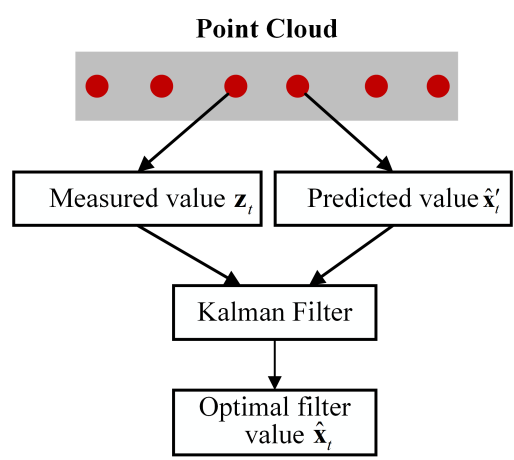

Fig. 5. Application of the Kalman filter to one point of a point cloud. 


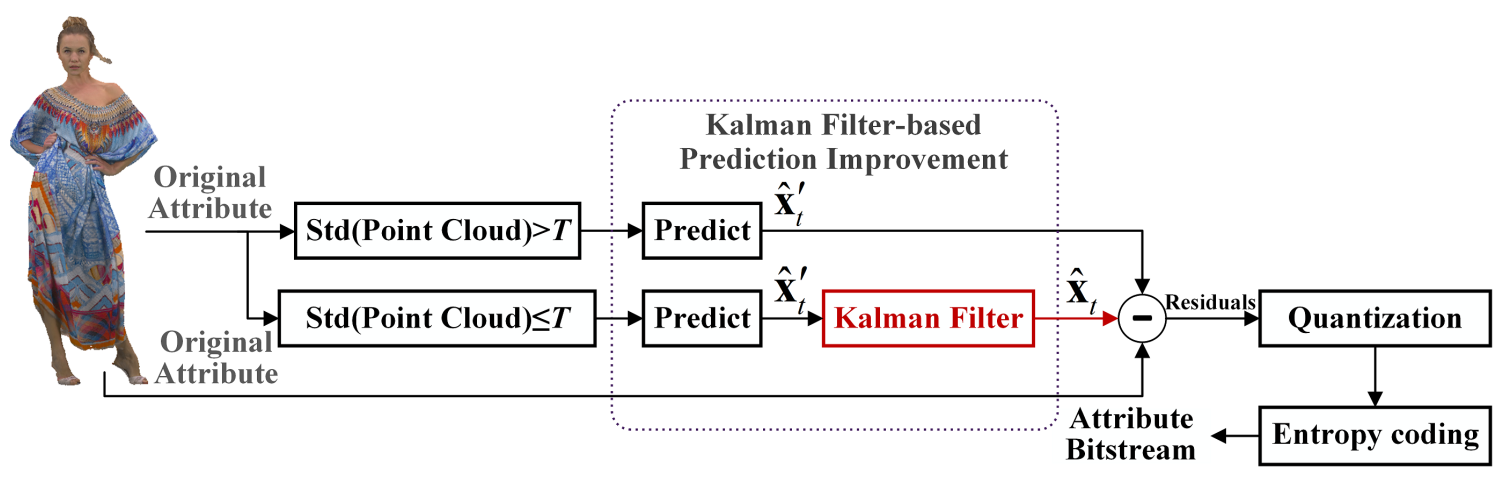

Fig. 6. System diagram of the proposed algorithm. Std(Point Cloud) denotes the standard deviation of the attributes in the R, G, B components. T is a threshold set empirically to 40 .

$\widehat{x}_{t}^{\prime}$ is calculated first as

$$
\boldsymbol{P}_{t}^{\prime}=\boldsymbol{A} \boldsymbol{P}_{t-1} \boldsymbol{A}^{T}+\boldsymbol{Q}
$$

where $\boldsymbol{P}_{t-1}$ is the prediction error covariance matrix of $\widehat{\boldsymbol{x}}_{\boldsymbol{t}-\mathbf{1}}$ and $Q$ is the covariance matrix of the process excitation noise whose initial value is set empirically. The Kalman gain $\boldsymbol{K}_{\boldsymbol{t}}$ at time $t$ can then be written as

$$
\boldsymbol{K}_{\boldsymbol{t}}=\boldsymbol{P}_{\boldsymbol{t}}^{\prime} \boldsymbol{H}^{T}\left(\boldsymbol{H} \boldsymbol{P}_{\boldsymbol{t}}^{\prime} \boldsymbol{H}^{T}+\boldsymbol{R}\right)^{-1}
$$

where $\boldsymbol{H}$ is the state observation matrix, which is usually the identity matrix, and $\boldsymbol{R}$ is the matrix of the inaccuracy of the system, whose initial value is set empirically.

b) Correction process: By using the measured value $z_{t}$ and the Kalman gain $\boldsymbol{K}_{t}, \widehat{\boldsymbol{x}}_{\boldsymbol{t}}^{\prime}$ can be refined as

$$
\widehat{\boldsymbol{x}}_{\boldsymbol{t}}=\widehat{\boldsymbol{x}}_{\boldsymbol{t}}^{\prime}+\boldsymbol{K}_{\boldsymbol{t}}\left(\boldsymbol{z}_{\boldsymbol{t}}-\boldsymbol{H} \widehat{\boldsymbol{x}}_{\boldsymbol{t}}^{\prime}\right),
$$

c) Update process: Finally, the error covariance is matrix updated as

$$
\boldsymbol{P}_{\boldsymbol{t}}=\left(\boldsymbol{I}-\boldsymbol{K}_{\boldsymbol{t}} \boldsymbol{H}\right) \boldsymbol{P}_{\boldsymbol{t}}^{\prime},
$$

where $\boldsymbol{I}$ denotes the identity matrix. The updated matrix $\boldsymbol{P}_{\boldsymbol{t}}$ is then used to filter the signal at time $(t+1)$.

\section{B. Kalman Filter-based prediction refinement}

We consider point clouds as discrete-time signals. The Kalman filter for one point in a point cloud is shown in Fig. 5. The reconstructed attribute of the previous point is used as the measured value $z_{t}$ of the current point, and the PT predicted attribute of the current point is taken as the prediction $\widehat{\boldsymbol{x}}_{\boldsymbol{t}}^{\prime}$ of the filter. Then, by calculating the Kalman gain $\boldsymbol{K}_{\boldsymbol{t}}$ of the current point and the measured value $z_{t}$, we can refine the prediction of the current point to get the filtered value $\widehat{x}_{\boldsymbol{t}}$, and then update the covariance matrix to calculate the Kalman gain $\boldsymbol{K}_{\boldsymbol{t}+\mathbf{1}}$ for the next point. The above operation is iterated until all points are traversed.

Fig. 6 shows a block diagram of the entire point cloud system. The following operations are based on the order of refinement levels (i.e., $R_{0}, R_{1} \ldots R_{N-1}$, where $N$ is the number of refinement levels), as shown in Fig. 1. To improve the results, we first determine whether the Kalman filter needs to be applied to the color component by checking its standard deviation. If the standard deviation is large (indicating the signals are not stationary enough), the efficiency of the Kalman filter will be reduced. In this case, we disable the Kalman filter. The standard deviation threshold is set empirically to 40. Because the number of points is very small in the first seven refinement levels $\left(R_{0} \sim R_{6}\right)$, the points of the first seven refinement levels are taken as a whole to calculate the standard deviation. For each subsequent refinement level (i.e., $R_{l}, l>6$ ), the same check is also done to decide whether to use the Kalman filter.

To further improve the results, we also directly transmit to the receiver the original attributes of a number of points from each refinement level. These points are selected as follows. If there are fewer than eight points in the refinement level, we select all of them. Otherwise, we select the eight points $P_{1}$, $P_{1+\lfloor M / 8\rfloor}, P_{1+2\lfloor M / 8\rfloor}, \ldots, P_{1+7\lfloor M / 8\rfloor}$, where the indices of the points correspond to the refinement level-based order, and $M$ is the number of points in this refinement level. This is because:

- The Kalman filter can produce more accurate results when the measured values are close to the original values.

- The transmitted original attribute can also be used as the reconstructed attribute to improve the quality of the reconstructed point clouds.

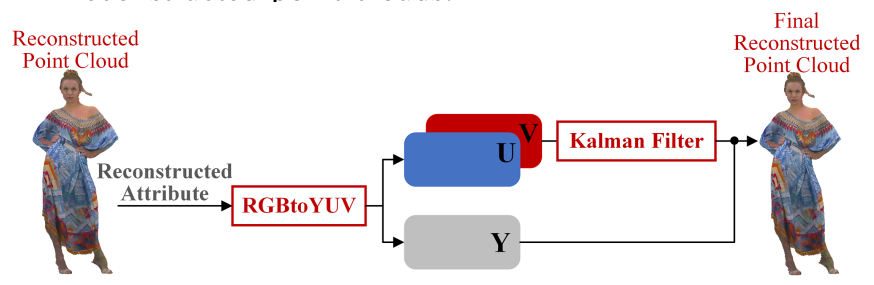

Fig. 7. System diagram of the proposed algorithm for improving the quality of the attribute of the reconstructed point cloud.

\section{Kalman Filter-based post-processing}

Furthermore, we also use a Kalman filter to enhance the quality of the reconstructed attributes. The system diagram is shown in Fig. 7. When the coding distortion is not large, the benefit of the Kalman filter is not obvious. Therefore, the proposed method is only used for quantization parameters $(Q P s)$ larger than or equal to 22 . As the texture values vary more in the luminance component than in the $\mathrm{Cb}$ and $\mathrm{Cr}$ 
components, the Kalman filter is only used for the $\mathrm{Cb}$ and Cr components.

During the Kalman filtering procedure, the average of the reconstructed attributes of the first three points is used as the measured value $z_{t}$, while the reconstructed attribute of the current point is used as the predicted value $\widehat{x}_{t}^{\prime}$. The filtering is used to filter the $\mathrm{Cb}$ and $\mathrm{Cr}$ components of the initial reconstructed attribute of the current point, while the $\mathrm{Y}$ component is left unchanged. The filtered value is used in the reconstructed point cloud.

\section{EXPERIMENTAL RESULTS}

We implemented the proposed methods in G-PCC test model TMC13 version 11.0 (TMC13V11) [16] and conducted experiments under the CY configuration of the common test conditions of MPEG G-PCC [17]. The computer used for the experiment has an Intel $17-8700 \mathrm{~K}$ processor and $32 \mathrm{~GB}$ RAM. Experiments were conducted for 21 point clouds from the Cat1-A and Cat1-B datasets provided by MPEG. To evaluate the performance, we used the Bjontegaard delta(BD)rate [18] and the complexity ratio. The $\mathrm{BD}$-rate gives the average bitrate reduction of the proposed method compared to TMC13V11 at the same reconstructed quality (a negative BD-rate corresponds to a reduction in bitrate). The complexity ratio is the ratio of the encoding time (resp. decoding time) of the proposed method to that of TMC13V11.

Table I compares the performance of the proposed Kalman filter-based prediction refinement to TMC13V11. We can see that an average $-0.46 \%,-2.82 \%,-3.64 \%$ BD-rate can be achieved for the Luma, Chroma $\mathrm{Cb}$, and Chroma $\mathrm{Cr}$ components, respectively. We can also see that the decoding complexity of the proposed method is slightly increased.

Table II shows the results for the proposed Kalman filterbased post-processing for point cloud quality enhancement. We can see that an overall average $-0.0 \%,-1.93 \%,-2.16 \%$ $\mathrm{BD}$-rate gain can be achieved for the Luma, Chroma $\mathrm{Cb}$, and Chroma Cr components, respectively.

Table III shows that combining the proposed two methods is advantageous as it allows to increase the average BD-rate gain to $-0.48 \%,-5.18 \%,-6.27 \%$ for the Luma, Chroma $\mathrm{Cb}$, and Chroma $\mathrm{Cr}$ components, respectively. Fig. 8 compares the visual quality of the reconstructed point clouds. We can see that the reconstruction of the clothes of loot_viewdep_vox 12 with the proposed methods is better than with TMC13V11.

As the Kalman filter is an optimal state estimation method [15], the refined prediction is more accurate than the original PT, and the reconstruction quality can also be improved by the Kalman filter-based post-processing. Since the luminance information is not stable enough, as the attribute values vary more in the luminance than in the $\mathrm{Cb}$ and $\mathrm{Cr}$ components, and the Kalman filter is more effective for stationary signals, the coding efficiency for the luminance is not as large as for the $\mathrm{Cb}$ and $\mathrm{Cr}$ components.

\section{CONCLUSIONS}

We proposed a Kalman filter-based prediction refinement technique and quality enhancement method for point cloud
TABLE I

AVERAGE BD-RATE GAIN OF THE METHOD PROPOSED IN SECTION III-B COMPARED WITH THE G-PCC TEST MODEL

\begin{tabular}{lccccc}
\hline \multirow{2}{*}{ Dataset category } & \multicolumn{3}{c}{ BD-rate for attributes (\%) } & \multicolumn{2}{c}{ Complexity Ratio(\%) } \\
\cline { 2 - 6 } & Luma & Chroma Cb & Chroma Cr & Encoding & Decoding \\
\hline Cat1-A average & -0.66 & -3.29 & -4.19 & 101 & 101 \\
Cat1-B average & -0.08 & -1.90 & -2.54 & 101 & 101 \\
Overall average & -0.46 & -2.82 & -3.64 & 101 & 101 \\
\hline
\end{tabular}

TABLE II

Average BD-RATE GAIN OF THE METHOD PROPOSED IN SECTION III-C COMPARED WITH THE G-PCC TEST MODEL

\begin{tabular}{lccccc}
\hline \multirow{2}{*}{ Dataset Category } & \multicolumn{2}{c}{ BD-rate for attributes (\%) } & \multicolumn{2}{c}{ Complexity Ratio(\%) } \\
\cline { 2 - 6 } & Luma & Chroma Cb & Chroma Cr & Encoding & Decoding \\
\hline Cat1-A average & 0 & -1.45 & -1.77 & 100 & 101 \\
Cat1-B average & 0 & -2.88 & -2.93 & 100 & 101 \\
Overall average & 0 & -1.93 & -2.16 & 100 & 101 \\
\hline
\end{tabular}

TABLE III

OVERALL AVERAGE BD-RATE GAIN COMPARED WITH THE G-PCC TEST MODEL.

\begin{tabular}{lccccc}
\hline \multirow{2}{*}{ Dataset Category } & \multicolumn{3}{c}{ BD-rate for attributes (\%) } & \multicolumn{2}{c}{ Complexity Ratio(\%) } \\
\cline { 2 - 6 } & Luma & Chroma Cb & Chroma Cr & Encoding & Decoding \\
\hline Cat1-A average & -0.69 & -5.31 & -6.54 & 101 & 102 \\
Cat1-B average & -0.08 & -4.94 & -5.72 & 101 & 102 \\
Overall average & -0.48 & -5.18 & -6.27 & 101 & 102 \\
\hline
\end{tabular}

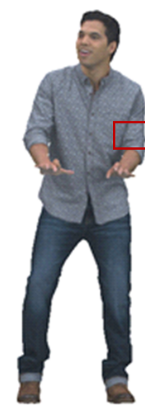

Original

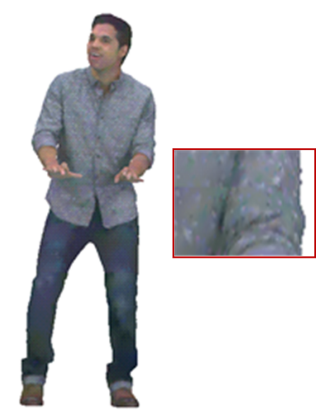

G-PCC Anchor

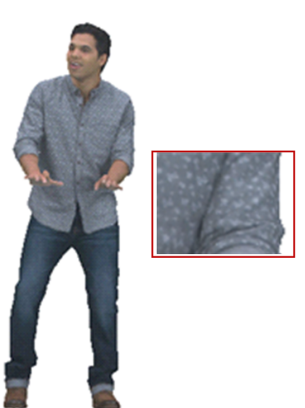

Our Method
Fig. 8. Visual quality comparison for loot_viewdep_vox12.

compression. In the prediction refinement method, the standard deviation of the attributes of the points to be encoded is calculated to decide whether using the Kalman filter is beneficial. In the quality enhancement method, only the Chroma $\mathrm{Cb}$ and Chroma $\mathrm{Cr}$ components are filtered when $Q P$ is large. Experimental results showed that $-0.48 \%,-5.18 \%,-6.27 \%$ BDrate gains can be achieved for the Luma, Chroma $\mathrm{Cb}$, and Chroma Cr components, respectively, compared with the stateof-the-art MPEG G-PCC test model.

\section{ACKNOWLEDGMENT}

This work has received funding from the National Natural Science Foundation of China under Grant 62172259 and 61871342, the major scientific and technological innovation Project of Shandong Province under Grant 2020CXGC010109, and the OPPO Research Fund. 


\section{REFERENCES}

[1] Y. Huang, J. Peng, C. C. J. Kuo and M. Gopi, "A generic scheme for progressive point cloud coding," in IEEE Transactions on Visual and Computational Graphics, vol. 14, no. 2, pp. 440-453, March-April 2008.

[2] T. Chen et al., "Layered projection-based quality assessment of 3D point clouds," in IEEE Access, vol. 9, pp. 88108-88120, 2021.

[3] E. S. Jang et al., "Video-Based Point-Cloud-Compression standard in MPEG: From Evidence Collection to Committee Draft [Standards in a Nutshell]," in IEEE Signal Processing Magazine, vol. 36, pp. 118-123, May 2019.

[4] ISO/IEC JTC1/SC29/WG7, "G-PCC codec description v9," document n0011, Oct. 2020.

[5] H. Liu, H. Yuan, Q. Liu, J. Hou and J. Liu, "A comprehensive study and comparison of core technologies for MPEG 3-D point cloud compression," in IEEE Transactions on Broadcasting, vol. 66, no. 3, pp. 701-717, Sept. 2020.

[6] R. L. de Queiroz and P. A. Chou, "Compression of 3D point clouds using a Region-Adaptive Hierarchical transform," in IEEE Transactions on Image Processing, vol. 25, no. 8, pp. 3947-3956, Aug. 2016.

[7] B. Kathariya et al., "Binary-tree based level-of-details generation for attributes coding in G-PCC," ISO/IEC JTC1/SC29/WG7, document m44940, Macao, CN, Oct. 2018.

[8] K. Mammou et al., "Lifting scheme for lossy attribute encoding in TMC1,” ISO/IEC JTC1/SC29/WG7, document m42640, San Diego, US, Apr. 2018
[9] S. Yea, A. Vosoughi, and S. Liu, "Bilateral filtering for predictive transform in G-PCC,” ISO/IEC JTC1/SC29/WG7, document m46365, Marrakech, Morocco, Jan. 2019.

[10] T. Sugio and C. Wang, "On improvement for adaptive reflectance predictor selection," ISO/IEC JTC1/SC29/WG7, document m43665, Ljubljana, SI, July 2018.

[11] C. Ma et al., "On filter based attribute prediction scheme," ISO/IEC JTC1/SC29/WG7, document m54951, Online, October, 2020.

[12] Q. Zhang et al., "Neighbors' weight modification on Lifting and Predicting Scheme," ISO/IEC JTC1/SC29/WG7, document m50773, Geneva, CH, Oct 2019.

[13] M. Alexa, J. Behr, D. Cohen-Or, S. Fleishman, D. Levin and C. T. Silva, "Computing and rendering point set surfaces," in IEEE Transactions on Visualization and Computer Graphics, vol. 9, pp. 3-15, Jan.-March 2003.

[14] Y. Lipman, D. Cohen-Or, D. Levin, and H. Tal-Ezer, "Parameterizationfree projection for geometry reconstruction, in ACM Transactions on Graphics, vol. 26, pp. 22-27, 2007.

[15] P. J. Hargrave, "A tutorial introduction to Kalman filtering," IEE Colloquium on Kalman Filters: Introduction, Applications and Future Developments, pp. 1/1-1/6, 1989.

[16] ISO/IEC JTC1/SC29/WG7, "G-PCC test model v11," document w19519, Jul. 2020

[17] ISO/IEC JTC1, "Common test conditions for point cloud compression," ISO/IEC JTC1/SC29/WG 07 MPEG 3D Graphics Coding, output document W19642, Oct. 2020.

[18] G. Bjontegaard, "Improvements of the bd-psnr model," VCEG-AI11, Berlin, Germany, Jul. 2008. 